\title{
América Latina y África. Entre la solidaridad sur-sur y los propios intereses
}

\author{
Latin America and Africa. Between South-South \\ solidarity and self-interests \\ Gladys Lechini*
}

\section{Resumen}

Este trabajo analiza las relaciones entre América Latina y África en el marco de la cooperación Sur-Sur a partir de tres casos particulares que muestran diferentes estrategias de inserción en el contexto de una política por impulsos. Brasil utilizó una diplomacia cultural y desarrolló una política acumulativa que dio sus frutos con la gestión de Lula, Argentina inició el acercamiento más temprano pero tuvo impulsos intermitentes y Venezuela, un actor tardío desplegó su petro-diplomacia con un discurso ideológico.

Palabras clave: América Latina, África, Brasil, Argentina, Venezuela, cooperación Sur-Sur

\section{Abstract}

This paper analyzes relations between Latin America and Africa within the context of South -South cooperation through three cases which show different strategies of an impulse-driven policy. Brazil has resorted to a cultural diplomacy, developing a cumulative policy that blossomed during Lula's

Profesora titular de Relaciones Internacionales, Universidad Nacional de Rosario (Argentina); Investigadora principal, CONICET (Argentina).gladystlechini@yahoo.com.ar. Recibido el 2 de abril de 2014; aceptado el 10 de octubre de 2014.

Se deja constancia de que parte del trabajo fue elaborada durante la estancia de la autora como Visiting Fellow del Stellnbosch Institute of Advanced Studies del Wallenberg Research Centre, Sudáfrica. 
Presidency. Argentina started the rapprochement earlier, but without continuity and Venezuela, a late newcomer, has developed its petro-diplomacy with an ideological rhetoric.

Keywords: Latin America, Africa, Brazil, Argentina, Venezuela, SouthSouth cooperation 
Gladys Lechini • América Latina y África. Entre la solidaridad Sur-Sur y los propios...

Las relaciones entre los Estados de América Latina y África -desde su respectiva independencia en los años sesenta- han mostrado un patrón de intermitencia que puede explicarse por los vaivenes propios de las situaciones internas y regionales, así como por los condicionantes sistémicos.

Si bien las dos regiones sufrieron el colonialismo europeo, las marcas de los colonizadores y las diferencias temporales en el acceso a la independencia, dejaron su impronta en la historia y la evolución sociopolítica y económica de sus respectivos países. La fuerte vinculación con los poderes centrales contribuyó a potenciar el desconocimiento mutuo y promover el olvido.

Los nuevos modos de inserción de estos Estados en el esquema global actual y la necesidad de promover alianzas alternativas entre países con condiciones similares y de buscar nuevos aliados en un contexto de diversificación de las relaciones internacionales favoreció los acercamientos. Estos fueron esporádicos, espasmódicos, pero a lo largo del tiempo posibilitaron tejer una malla de relaciones gubernamentales y privadas, que aunque débil, está presta a ser fortalecida en coyunturas favorables.

Con este marco, el trabajo apunta a realizar una breve introducción a las relaciones interregionales, con énfasis en el presente siglo, discutir la cooperación Sur-Sur desarrollada entre estos Estados y avanzar en la descripción y análisis de tres casos particulares de relaciones entre América Latina y África: Brasil, Argentina y Venezuela.

\section{BREVE HISTORIA DEL AVANCE DE LA COOPERACIÓN ENTRE LAS REGIONES}

América Latina y África pudieron haber estado encastradas cuando existió Gondwana, en el triásico, pero se separaron en tiempos remotos al iniciarse la deriva de los continentes hasta su actual posicionamiento. Este alejamiento perduró hasta que ambas regiones fueron "descubiertas" por el europeo y se reencontraron a través del tráfico de esclavos. Fueron los propios colonizadores quienes, sin quererlo, sentaron las bases de una conexión cultural que perduraría a través de los años, al promover el ignominioso tráfico de esclavos que se inició en el siglo XVI.

Los portugueses y españoles que llegaron a la América Latina se diferenciaron de los ingleses, franceses, belgas y holandés en que se establecieron en África. El español y el portugués fueron y son las lenguas de la América Latina (con excepción de las pequeñas colonias del Caribe y las Guyanas). El continente africano se dividió de acuerdo con las lenguas del colonizador europeo que salió triunfante de las guerras mundiales: anglófonos, francófonos, lusófonos.

A lo largo de los siglos y a pesar de cierta afonía, hubo períodos en que la conexión transatlántica fue expresiva, aunque en sus orígenes haya tenido un fuerte contenido esclavista. Por ejemplo, los contactos entre las colonias portuguesas de América del Sur y África posibilitaron un comercio intra- 
sudatlántico importante, que se mantuvo hasta la independencia de Brasil en 1922 (Sombra Saraiva, 1996). En el caso de Argentina, la entonces colonia española de Fernando Póo y Annobón, posteriormente Guinea Ecuatorial, al momento de su creación dependía del Virreinato del Rio de la Plata, en $1776^{1}$.

En el siglo XIX, con la independencia de las colonias latinoamericanas de sus metrópolis ibéricas, la relación con las colonias africanas fue triangulada a través de Europa y por tanto, de baja intensidad. La situación comenzó a cambiar en la segunda postguerra con las independencias africanas y el posterior acceso de sus Estados al sistema de las Naciones Unidas, punto de encuentro con los representantes latinoamericanos.

La descolonización de Asia y África marcó el surgimiento del Sur como actor internacional, concretado en reuniones como la Conferencia de Bandung en 1955, la conformación del Movimiento de Países No Alineados en 1961 y la creación en Ginebra del Grupo de los 77 (G77) en 1964 -en el marco de la reunión de la primera Conferencia de las Naciones Unidas sobre Comercio y Desarrollo (UNCTAD).

En este contexto, el Sur periférico surgió marcado por la ideología de la

Según documenta Rizzo Romano (1991) "en los legajos de la División Colonia, Sección Gobierno, Isla Fernado Póo, constan cinco voluminosos paquetes de documentos correspondientes al período 1778 1794, referidos a la administración, desde Buenos Aires, de esos territorios africanos" liberación nacional, contra el colonialismo, sosteniendo los principios de la no injerencia en sus asuntos internos y con el objetivo de lograr el desarrollo económico mediante la eliminación de las asimetrías con el Norte, dando prioridad a las asociaciones y coaliciones entre sí.

La idea de una alianza del Sur se vio reflejada en el espíritu de la reunión que tuvo lugar en La Habana, en 1966, con la organización de la Primera Conferencia Tricontinental que creó la Organización de Solidaridad con los Pueblos de América Latina, Asia y África (OSPAAAL) para promover la solidaridad con los reclamos y las luchas de los pueblos del Tercer Mundo (Zuluaga Nieto, 2006). Para ese entonces se hablaba del Trueno de Bandung, del surgimiento del Tercer Mundo como nueva fuerza vital en los asuntos internacionales (Worsley, 1972).

Con la independencia de los nuevos Estados africanos y el reconocimiento de su status internacional y soberano en las Naciones Unidas, se produjo el primer acercamiento entre América Latina y África, a través de las relaciones establecidas en la Asamblea General junto al Grupo Latinoamericano (GRULA), que apoyaba los procesos de liberación nacional. Se enviaron representantes para asistir a las celebraciones de la independencia de los distintos países y las primeras misiones exploratorias (Lechini,1986).

Pero fue en la década de los años setenta que la cooperación entre los países del Sur pareció posible a través 
Gladys Lechini • América Latina y África. Entre la solidaridad Sur-Sur y los propios...

de acciones que apuntaban a mostrar un poder conjunto para cambiar un orden injusto. Se confiaba en que la cooperación entre quienes sufrían las mismas situaciones de dependencia posibilitaría reforzar su capacidad de negociación con el Norte. Ese potencial se vio reflejado con el shock petrolero de 1973 y sus efectos en las economías de los países desarrollados, mostrando las posibilidades de una concertación entre países productores de materias primas; y en 1974, cuando la Asamblea General de Naciones Unidas aprobó la Declaración de un Nuevo Orden Económico Internacional (NOEI) y la Carta de los Derechos y Deberes Económicos de los Estados.

Los encuentros multilaterales habilitaron los contactos bilaterales iniciándose un proceso de conocimiento mutuo. La iniciativa de los acercamientos -que denominamos impulsos- provino de los países latinoamericanos que ya eran actores interesados en relacionarse con los nuevos Estados africanos, buscando acercar posiciones en los espacios multilaterales. Esta aproximación reflejó variados modelos que respondieron a las propias características de los países y a los objetivos de los impulsos. En Latinoamérica los impulsos se sustentaban en razones ideológicas (Cuba), comerciales (Brasil) o buscando apoyo en las votaciones que tenían lugar en los espacios globales (Argentina).

En el caso africano fue Sudáfrica el país que tomó iniciativas para promover los contactos cuando el gobierno del Apartheid desarrolló estrategias para impedir un mayor aislamiento internacional y conseguir el apoyo de las dictaduras latinoamericanas a su proyecto racista (Lechini,1995). Por el contrario, a partir de 1994 y el ascenso de un gobierno democrático multirracial bajo la presidencia de Nelson Mandela, el objetivo fue reinsertarse en el escenario global junto a los países del Sur (Barber,2004).

En los años setenta, frente a los problemas económico-financieros de los países del Norte y el proceso de distensión entre las dos superpotencias, los países del Sur creyeron que podrían revertir una situación internacional que los perjudicaba y modificar la agenda impuesta por el Norte. Sin embargo, la práctica demostró lo ilusorio de las expectativas. Si bien los países en desarrollo tenían problemas similares, la complejidad de su situación interna les impidió encontrar soluciones comunes y la tentación de las salidas individuales primó por sobre cualquier naciente solidaridad Sur-Sur.

En los años ochenta, la crisis de la deuda ofreció otra buena oportunidad para desarrollar acciones coordinadas. No obstante, las políticas implementadas por los Estados desarrollados junto a los acreedores privados, sumadas a las fragilidades económicas de los países endeudados, disolvieron las tentativas de cooperación multilateral. A pesar de ello, los gobiernos latinoamericanos que habían recuperado la democracia fueron capaces de implementar políticas de concertación para la resolución de conflictos en la región (Consenso de 
Cartagena, Grupo de Contadora, Grupo de Apoyo a Contadora, Grupo de los 8). Asimismo, promovieron nuevos acercamientos bilaterales con sus pares africanos, con el afán de reconstruir la cooperación Sur-Sur.

En los años noventa, la finalización de la Guerra Fría, el avance de la globalización, la implementación de las políticas neoliberales y los graves problemas económicos de los países en desarrollo diluyeron el accionar multilateral del Sur, que se había gestado a partir del diseño y aplicación de políticas gubernamentales. Aceptar las reformas neoliberales significó para los países del Sur que el Estado, que originariamente había sido el agente primario del desarrollo, fuera ahora el problema.

El siglo XX concluyó con los países del Sur sufriendo las consecuencias negativas derivadas de la aplicación de las medidas impuestas por el llamado Consenso de Washington, que agudizaron la brecha entre ricos y pobres, dejando en el propio Sur una pesada deuda social. Los países de América Latina se vieron inmersos en complejas situaciones internas, en tanto que África había sido presa del "Afropesimismo", la "fatiga de los donantes" y el olvido.

A pesar de todos los problemas y presagios negativos, el siglo XXI trajo renovadas esperanzas para la cooperación Sur-Sur entre estas regiones, a medida que se avanzaba en las relaciones bilaterales y en paralelo se promovía el multilateralismo (en las Naciones Unidas y la Organización Mundial del Co- mercio), el minilateralismo (IBSA- G20 comercial y G20 financiero ${ }^{2}$ ) y el interregionalismo (ASPA, ASA,ZPCAS ${ }^{3}$ ).

2 El Foro IBSA (India, Brasil y Sudáfrica) y el G20 comercial fueron creados en 2003, en tanto el G20 financiero está conformado por Estados desarrollados y emergentes, surgió a finales de los noventa para tratar los problemas financieros globales y se reflotó con la crisis financiera de fines de la primera década del siglo XXI

3 Los encuentros entre los Estados de América del Sur y los Países Árabes (ASPA) incluyen a 10 africanos: Argelia, Comoras, Djibouti, Egipto, Libia, Marruecos, Mauritania, Somalia, Sudán y Túnez. Se reunieron en 2005 en Brasilia, Brasil, en 2009 en Doha, Qatar y en 2013, en Lima, Perú. La primera cumbre de países de América del Sur y África (ASA) tuvo lugar en Abuja, Nigeria en 2006; a ella le siguieron las reuniones de Isla Margarita, en Venezuela, en 2009 y en Malabo, Guinea Ecuatorial, en 2013. Asimismo se produjo el relanzamiento de la Zona de Paz y Cooperación del Atlántico Sur en Luanda, Angola, en 2007 y luego en Montevideo, Uruguay en 2013. Sus miembros son Argentina, Angola, Benin, Brasil, Camerún, Cabo Verde, Congo, Costa de Marfil, Guinea Ecuatorial, Gabón, Gambia, Ghana, Guinea, Guinea-Bissau, Liberia, Namibia, Nigeria, República Democrática del Congo, Santo Tomé y Príncipe, Senegal, Sierra Leona, Sudáfrica, Togo y Uruguay. La ZPCAS surgió en 1986 para promover la cooperación y el mantenimiento de la Paz y Seguridad en el Atlántico Sur y de esta manera prevenir la creciente militarización de la región y la posible injerencia de potencias extra-regionales. La ZPCAS pasó por varios períodos, resultado tanto de los cambios sistémicos como de los procesos políticos y económicos internos que vivían los países a ambos lados del Atlántico, "aggiornando" y modificando sus objetivos. Desde su creación se han 
Gladys Lechini • América Latina y África. Entre la solidaridad Sur-Sur y los propios...

\section{La coOperación Sur-Sur (CSS)}

Como se señaló, el proceso de acercamiento entre los países de América Latina y África se ha desarrollado en el contexto de las relaciones Sur-Sur (Delgado Caicedo y Barrera, 2010), lo cual conlleva realizar algunas precisiones respecto a esta noción. El concepto "Sur" desborda la categoría geográfica y se asienta en una concepción política y económica. Los países del Sur son países en vías de desarrollo, periféricos, que comparten desafíos y situaciones de vulnerabilidad. Sin embargo, no son un grupo homogéneo debido a sus diferencias socioeconómicas y políticoculturales, tanto dentro de su contexto local como en el ámbito regional.

Sin embargo, pueden encontrarse varias similitudes. La idea del Otro como ser colonizado (Said,1996) es de alguna manera uno de los conectores entre los países del Global South, como se lo está denominando actualmente Ambas subregiones fueron colonias europeas y debieron luchar activamente para obtener su independencia. Poseen relaciones dependientes y asimétricas con los países centrales y sus economías sufrieron los condicionamientos de los programas de ajuste estructural dictaminados por las instituciones fi-

realizado las siguientes reuniones ministeriales: en Brasilia (1988 y 1994); en Abuja, Nigeria (1990); en Sommerset West, Sudáfrica (1996); en Buenos Aires (1998) y las ya mencionadas en Luanda (2007) y Montevideo (2013). nancieras internacionales, si bien ellos comenzaron a aplicarse en África en los años ochenta y en América Latina en los años noventa. En la actualidad atraviesan por situaciones de aprendizaje y lucha por lograr estabilidad política y desarrollo económico en un contexto en que las reglas del juego no les son muy favorables.

La idea de promover la cooperación entre los países del Sur surgió como complemento y a la vez alternativa a la relación asimétrica Norte-Sur. Como el concepto de Cooperación Sur- Sur (CSS) es muy elástico y elusivo, en función de los variados contextos en que ha sido utilizado, corresponde realizar algunas especificaciones. En sentido amplio, la CSS puede ser entendida como "coalition building", es decir como cooperación esencialmente política. En sentido más restringido, acercándose a las definiciones utilizadas por el Programa de las Naciones Unidas para el Desarrollo (PNUD), que la interpreta de manera más pragmática, concreta y específica, como cooperación horizontal o cooperación técnica entre países en desarrollo.

La cooperación Sur-Sur se diferencia de las relaciones Sur-Sur, en la medida en que estas últimas se despliegan naturalmente entre los varios actores públicos y privados de ambas regiones. En tanto, la CSS implica una construcción esencialmente política que apunta a reforzar las relaciones bilaterales y a formar coaliciones en los foros multilaterales, para obtener mayor poder de negociación conjunto. Se basa en el su- 
puesto de que es posible crear una conciencia cooperativa que les permita a los países del Sur afrontar los problemas comunes mediante el fortalecimiento de su capacidad de negociación con el Norte y de la adquisición de mayores márgenes de maniobra en el plano internacional. Por su propia naturaleza, requiere de ciertos supuestos básicos comunes (likemindedness), que se explicitan en las negociaciones diplomáticas pero también que se complementan con variadas actividades como el fomento del comercio, la asistencia técnica, entre otras (Lechini, 2009).

Sin embargo, en muchas oportunidades se ha utilizado retóricamente el discurso de la CSS en ámbitos multilaterales y en visitas y encuentros bilaterales, como parte de una "puesta en escena”, pero con baja intencionalidad. Las propias realidades internas y los múltiples y variados intereses nacionales y locales no siempre marchan en la misma dirección de las "comunalidades" expresadas en los discursos oficiales.

La CSS también puede entenderse según los espacios geográficos, los actores involucrados y las dimensiones específicas. En términos geográficoespaciales, puede producirse cooperación Sur-Sur en el ámbito regional (por ejemplo, entre los países de América del Sur), en el contexto interregional (la que se desarrolla entre los países de América del Sur y África) o transregional (como la asociación entre India, Brasil y Sudáfrica -IBSA).
En cuanto a los actores involucrados, la cooperación Sur-Sur es básicamente realizada por los Estados, pero también muchas veces son relevantes los aportes de actores no gubernamentales al desarrollo de sus programas y proyectos.

En cuanto a sus dimensiones se destacan la cooperación técnica y científico-tecnológica, la económicacomercial, la académica y la diaspórica. La llamada cooperación horizontal entre Estados de similar desarrollo generalmente se relaciona con la cooperación técnica y científico-tecnológica ${ }^{4}$. La primera alude a la transferencia de capacidades técnicas y administrativas (el know-how o saber-hacer) entre los propios países en desarrollo, en tanto la segunda se orienta a la realización de actividades conjuntas de investigación para promover avances científico- tecnológicos para un desarrollo integral (Costa Leite, 2012). Como ejemplos cabe citar la cooperación técnica desarrollada por Argentina y Brasil con los países africanos en la década de los años ochenta o las actividades del Fondo Argentino de Cooperación Horizontal

4 En la década de los años setenta se comenzó a producir una interacción entre los países del Sur posibilitando la trasmisión de experiencias positivas. Por entonces, las Naciones Unidas promovieron el concepto de "cooperación técnica entre países en desarrollo" (CTPD), creando en 1974 una unidad especial en el ámbito del PNUD. En 1978 se organizó en Argentina la primera Conferencia de Naciones Unidas sobre CTPD, cuyas recomendaciones fueron aprobadas en el Plan de Acción de Buenos Aires. 
Gladys Lechini • América Latina y África. Entre la solidaridad Sur-Sur y los propios...

(FOAR) -dependiente de la Dirección General de Cooperación Internacional en Argentina- y de la Agencia Brasilera de Cooperación (ABC) en Brasil. (Malacalza, 2013). En esta línea se considera la llamada cooperación en cascada, ya que los países relativamente más desarrollados -que han adaptado las tecnologías de los países industrializados a sus propios territorios y necesidades- las transfieren a sus socios del Sur, tal como lo hizo Brasil con su tecnología tropicalizada 5 .

También en este ámbito vale mencionar las contradicciones que se presentan entre discurso y práctica, entre compromisos externos y demandas internas. En situaciones en que al interior de los países latinoamericanos hay una gran deuda social, con importantes sectores de población marginal, cuesta mucho explicar y justificar los fondos que se dedican a la cooperación internacional, aun cuando sea en el contexto Sur-Sur.

Por otra parte, la cooperación económica-comercial entre los países del Sur se sustenta en la toma de conciencia por parte de los gobiernos de su situación desventajosa en las negociaciones para acordar un régimen internacional de comercio e inversiones. Ello explica la búsqueda de alternativas a través de la formación de coaliciones o alianzas

Tecnología importada de los países desarrollados del norte y adaptada a las necesidades y condiciones sociales y climáticas de los países situados en los trópicos, apta para utilizarse en áreas selváticas o con altas temperaturas intergubernamentales en las negociaciones multilaterales -tanto dentro como fuera de la Organización Mundial del Comercio (OMC)- para sentar las bases de una nueva configuración, más justa y equitativa, del poder mundial (Keet,2006).

La cooperación académica cobró nuevo impulso cuando las corrientes de análisis acuñadas en el Norte, eurocéntricas y lineales, no dieron cuenta de los problemas que aquejan a los países del Sur. El modelo impuesto en la década de los años noventa generó en los países de América Latina una direccionalidad muy marcada en los trabajos académicos marginando opciones como la africana, por ser investigaciones ociosas, impertinentes, débiles o inútiles. Dos factores contribuyeron a consolidar esta tendencia: un esquema de vinculación entre conocimiento y poder, esto es, "produzcamos conocimiento para los espacios de poder vigentes", y la condicionalidad de las principales líneas de financiamiento. Con esta particular orientación se cerraron las puertas para habilitar nuevos modos de pensar la inserción internacional de nuestros países en el Sur. Por ello hoy se plantea descolonizar

las ciencias sociales (Lander,2000) para modificar la forma de producción de conocimiento y poder transformar las relaciones de poder.

La presencia africana en América, y en particular en algunos países caribeños y sudamericanos como Colombia, Venezuela y Brasil, ha dejado huellas indelebles en la cultura y conformación 
de los Estados nacionales. Consciente de ello, la Unión Africana ha designado a la Diáspora como la sexta región de África, en reconocimiento a la vital contribución que ella puede hacer al desarrollo, tal como lo planteó Marcus Garvey cuando sostuvo que África era para los africanos del continente y del exterior. La confluencia de estas dos caras hace de la cooperación diaspórica otro componente vital de la cooperación Sur-Sur entre América Latina y África, como lo demostró la primera conferencia global sobre la diáspora que tuvo lugar en Barbados en 2007.

\section{LOS IMPULSOS BILATERALES}

A nivel bilateral las relaciones entre América Latina y África han ido creciendo y fortaleciéndose a partir de visitas mutuas de alto nivel, del incremento del comercio y de la cooperación científico-técnica, con diferentes tiempos y “marcas de origen”. En general, el patrón de las relaciones de los Estados latinoamericanos con los africanos está marcado por la dinámica de los impulsos generados desde la costa occidental del Atlántico, con objetivos político-diplomáticos, comerciales o estratégico-militares.

La mayoría de las veces estos impulsos generaron una relación intermitente, como puede verse en el caso de los acercamientos argentinos, pues el conjunto de acciones no generó una masa crítica que promoviera el diseño de estrategias para los Estados de África. Los impulsos fueron aislados, discontinuos, respondiendo a iniciativas puntuales y a un proceso de gestación de iniciativas de carácter más bien "individual”. En el caso de Brasil, también se produjeron impulsos, pero las acciones políticas brasileñas fueron acumulativas, permitiendo la construcción, con altos y bajos, de una política africana. El caso de Venezuela también es muy especial, pues después de muchos años de mirar hacia Estados Unidos, con Chávez en el gobierno se redescubrió la africanía por las venas venezolanas y se decidió promover el acercamiento a los países de la región, con un potente discurso y acciones impulsivas, amparadas en la petrodiplomacia.

Brasil y los avances en la formulación de una política africana

Aunque Brasil contaba con una historia colonial común plena de contactos intra-sudatlánticos, luego de su independencia y por imposición de Portugal se vio impedido de continuar las relaciones con las colonias portuguesas en África. Este alejamiento involuntario comenzó a revertirse en el siglo XX, gracias a un proceso de recomposición gradual de relaciones con los nuevos Estados africanos, en el marco de su política atlántica.

En Brasil, el acercamiento político diplomático estuvo vinculado a las estrategias presidenciales y su principal agente fue el Ministerio de Relaciones Exteriores, aunque también hubo participación variable de la corporación militar, así como de empresarios, parlamentarios y académicos en lo que 
Gladys Lechini • América Latina y África. Entre la solidaridad Sur-Sur y los propios...

de Souza (2002:26) denomina la "comunidad de política externa".

La aproximación al África fue una cuestión de principios e intereses, para promover el desarrollo de la solidaridad entre los países "del Sur" y garantizar una presencia internacional que aumentase la capacidad de influencia del país en cuestiones globales, a partir de una mayor diversificación de sus relaciones externas y de alianzas -tanto políticas cuanto económicas.

A medida que avanzaba la construcción diplomática se utilizó el discurso culturalista que enfatizaba la familiaridad y la historia común a ambos lados del Atlántico (Saraiva, 1996), y la denominada diplomacia cultural (Mourao, 1994) para movilizar a la sociedad y promover el comercio. La influencia africana en Brasil es sumamente relevante desde el punto de vista étnico y cultural conformando parte del corpus social brasileño. Como dijera Gilberto Gil, cantante brasileño y ministro de cultura de Lula da Silva (2003-2008): entre Brasil y África hay una conexión cultural, estética y genética ${ }^{6}$.

La política africana de Brasil fue una política de lo posible, que tuvo importantes condicionantes externos, especialmente en la vinculación con las colonias africanas de Portugal, país con el cual Brasil mantenía una relación especialísima. Por ello hubo ambigüedades en las relaciones con las colonias portuguesas en África hasta que se optó por la opción africana, con

6 Back 2 Black Festival, Rio de Janeiro, 28 de agosto de 2009 el reconocimiento de sus independencias. También hubo ambigüedades con Sudáfrica, dado que las oscilaciones de Brasil traducían intereses. En la relación bilateral, hasta mediados de la década de los años setenta Brasilia dio respuesta a los impulsos sudafricanos, que fueron entendidos como ambigüedades (Vilalva y Gala, 2001: 55), vacilaciones (Penna, 2001) o contradicciones (Saraiva, 1996). Pero las relaciones con el gobierno del apartheid disminuyeron a medida que se consolidaban los vínculos políticos y económicos con los Estados del África subsahariana. En tanto, con la nueva democracia multirracial en Sudáfrica, Brasil inició un inequívoco proceso de construcción de alianzas estratégicas.

Los diferentes gobiernos que se sucedieron en Brasil fueron construyendo una política africana hasta que en los años noventa el acercamiento fue más selectivo debido a condicionantes externos e internos y a una limitación de los recursos. Como contrapartida, o quizás de manera reactiva, con la llegada al gobierno del presidente Luiz Inácio Lula da Silva se retomó y reforzó la política africana mediante un proceso de intensos acercamientos diplomáticos y relaciones comerciales fructíferas (Lechini,2006:103-138).

El primer impulso lo dio el gobierno de Janio Quadros iniciado, en 1961, en el marco de la llamada "política externa independiente", donde por primera vez surgió una estrategia política global para África, que incluyó la apertura de embajadas en varios países africanos. 
Pero esta iniciativa perdió fuerza con el golpe militar que trajo la línea pro occidental de Humberto Castello Branco (1964-1967)

Sin embargo, con Arthur Costa e Silva (1967-1969), la política africana fue redefinida e Itamaraty se tornó conductor de los objetivos de la diplomacia de la prosperidad, en tanto con Emilio Garrastazu Médici (1969-1974) se redimensionó la política africana, aunque las disputas intra-burocáticas mostraron la ausencia de consenso sobre el rol de África en las definiciones estratégicas. Fue en 1972 que el ministro Gibson Barboza presidió la primera misión que visitó varios países africanos de la costa occidental, constituyéndose en fundacional para el posterior desarrollo del acercamiento brasileño.

En términos generales, tanto Emilio Garrastazu Médici como su sucesor Ernesto Geisel (1974-1979) mantuvieron una política exterior destinada a reducir el grado de dependencia de Brasil. Por entonces, el pragmatismo ecuménico y responsable formulado por este último se tornó un instrumento para la acción externa de Brasil, iniciándose un periodo de diez años de muy buenas relaciones con los Estados africanos. En este período prolífico en contactos, la densidad de las relaciones político-diplomáticas se vio reflejada en la apertura de nuevas embajadas, en el alto nivel de las visitas y de las misiones enviadas a través del Atlántico, y en la concreción de acuerdos bilaterales de cooperación comercial y técnica. A su vez, aumentaron las rutas de comercio en el Atlántico y las inversiones en proyectos de desarrollo.

Aunque la aproximación al África pudo haber sido una estrategia política, la dimensión comercial no estuvo ausente y fue, de hecho, la parte más visible. En los años setenta, el comercio exterior había asumido una importancia creciente en función del modelo de desarrollo económico, que requería importar petróleo y bienes de equipamiento. África aparecía como un mercado potencial, en el marco de la cooperación Sur-Sur naciente y de la estrategia de inserción internacional de Brasil. La vulnerabilidad energética brasileña fue un estímulo para la aproximación a los Estados africanos productores de petróleo. Como resultado, las relaciones comerciales presentaron una concentración muy acentuada en pocos países.

El aumento del intercambio no se dio únicamente en los aspectos puramente comerciales, sino también en lo que se refiere a la venta de servicios, tecnología y bienes de capital destinados a obras de infraestructura: construcciones de represas hidroeléctricas, autopistas, transportes ferroviarios, obras de saneamiento, telecomunicaciones, agropecuarias, e incluso capacitación de personal técnico.

Si bien las crisis petroleras de 1973 y 1979 y la crisis de la deuda externa de 1982 constituyeron ataques al modelo defendido por Brasil, con João Figueiredo (1979-1985) se mantuvo aún el énfasis en la política de aproximación al continente africano, derivada del 
Gladys Lechini • América Latina y África. Entre la solidaridad Sur-Sur y los propios...

"universalismo" implementado por el canciller Ramiro Saraiva Guerreiro. Con los conceptos de doble inserción en Occidente y en el Tercer Mundo vía la cooperación Sur-Sur, se apuntaba a sumar estrategias y no excluir campos antagónicos.

Por esto, las relaciones políticas de Brasil con África durante su gobierno ascendieron a un nivel de densidad relevante, constituyendo su viaje al África en 1983 -abarcando países lusófonos, francófonos y anglófonos- el primero de un presidente brasileño y sudamericano. Esta visita se constituyó en el mejor aval del interés brasileño por los países africanos y un momento muy alto en la construcción de la política africana. Como contrapartida, en este período Brasil recibió innumerables visitas de cancilleres y jefes de Estado de África, demostrando el interés recíproco afro-brasileño ${ }^{7}$.

El período del presidente José Sarney (1985-1990) beneficiado por el ambiente democrático instaurado por la Nueva República, siguió los trazos de la política africana ya delineados, con los límites impuestos por la negociación de la deuda externa y la inestabilidad económica interna. Consecuentemente hubo una disminución del perfil y comenzó a avanzar el selectivismo. El mayor acercamiento a Angola y a los

Tanto es así que para fines del gobierno de Figueiredo se mantenían relaciones diplomáticas con 43 de los 52 países africanos, con 21 embajadas residentes en África y con 13 embajadas de países africanos en Brasilia

países del África austral, sumado al incremento de la tensión en Sudáfrica, llevaron al presidente a tener una posición más crítica frente a Pretoria tanto a nivel del discurso en las Naciones Unidas como en la práctica, tomando medidas concretas como el llamado decreto Sarney ${ }^{8}$, que se convirtió en guía de la relación con Sudáfrica hasta el final del régimen racista blanco.

Por entonces, la preocupación por la seguridad militar en la región sudatlántica, por la participación de potencias extra-regionales y la crisis sudafricana, llevaron a Brasil a proponer la creación de una zona de Paz y Cooperación en el Atlántico Sur (ZPCAS). Pero la seguridad no fue el único leit motiv de la iniciativa brasileña. Itamaraty necesitaba darle nuevo ímpetu a su política africana y tomaba medidas innovadoras para neutralizar las desventajas de estar posicionado lejos de los principales centros económicos y compensarlo con un mayor comercio intrarregional.

Durante los años noventa, el incremento de la vulnerabilidad externa trajo aparejados ajustes a la política africana, acentuándose la selectividad, ahora encubierta por la persistencia del recurso a la diplomacia cultural. Las relaciones bilaterales comenzaron a ser

8 Este Decreto No 91524, del 9 de agosto de 1985, reunió prohibiciones nuevas con otras anteriores. Prohibía el intercambio cultural, artístico y deportivo; la exportación de petróleo y combustibles derivados; el abastecimiento y transporte de armas y material análogo de cualquier tipo. 
puntuales, con aquellos países en condiciones de responder a las necesidades brasileñas, incluyéndose, en los casos en que fue políticamente necesario, la idea de vinculación entre asociaciones regionales.

Sudáfrica -ya democrática- obtuvo atención creciente, junto a Angola y Nigeria; en el ámbito multilateral se promovió la cooperación con los países de la Comunidad para el Desarrollo del África Austral (SADC) bajo el paraguas del MERCOSUR, con la Comunidad de Países de Lengua Portuguesa (CPLP) ${ }^{9}$ y con la Zona de Paz y Cooperación del Atlántico Sur $(\mathrm{ZPCAS})^{10}$, participando en las Operaciones de Mantenimiento de Paz en África. Los viajes presidenciales y las visitas de alto nivel muestran la dirección de las opciones ${ }^{11}$, tal como los convenios y acuerdos firmados con

9 Brasil contribuyó a la creación de la CPLP en 1996, como un nuevo mecanismo de cooperación entre los países luso-parlantes, con los africanos Angola, Mozambique, Guinea Bissau, Cabo Verde y Sao Tome e Príncipe

10 En 1992 Brasil volvió a dar impulso a la idea de la cooperación en el Atlántico, la cual había sido desvalorizada con el fin de la guerra fría, incorporándole ahora a la ZPCAS nuevos objetivos.

11 en septiembre de 1991, Collor de Mello visitó cuatro países del África Austral: Namibia, Angola, Mozambique y Zimbabwe. El ministro de Relaciones Exteriores de Itamar Franco, Celso Amorím, asistió a la toma de posesión del presidente Mandela. El ministro Lampreia visitó Sudáfrica en tres oportunidades (1995, 1996 y 2000) y fue a Angola (1998) y a Mozambique para la Tercera Reunión de la CPLP (2000), acompañando al presidente.
Sudáfrica, Namibia, Angola, Mozambique, Cabo Verde, Santo Tomé y Príncipe y Nigeria.

Esta disminución del énfasis en la política africana y el avance de la selectividad pueden explicarse con el programa de modernización liberal de Fernando Collor de Mello (1990$1992)^{12}$ que se acentuó durante la presidencia de Fernando Henrique Cardoso, sustituyéndose la tradicional visión "desarrollista" de la política exterior brasilera por un nuevo ideario neoliberal (Lampreia, 1995) de acuerdo con el cual acercarse al Tercer Mundo era obsoleto y anacrónico.

Como las limitaciones económicofinancieras fueron un constreñimiento importante, Itamaraty intentó privilegiar la aproximación política, a partir de la propuesta de un tratado de desnuclearización y de protección del medio ambiente del Atlántico Sur, en el marco de la ZPCAS, como manera creativa y útil de mantener a África en su agenda de política exterior. Asimismo, la convocatoria a sectores privados para participar en las cuestiones externas y el retorno a la democracia en Brasil fueron cambios que favorecieron a las comunidades de afrodescendientes y de grupos pro África ${ }^{13}$ que ya con Sarney habían conseguido hacer oír su voz pidiendo

12 Con Itamar Franco el neoliberalismo se atenuó y hubo una vuelta tímida a la política africana

13 Prueba de ello son el surgimiento en 1996 del Colectivo de Empresarios Afro-brasileños (CEABRA), y en 1999 del Frente Parlamentario Brasil-África. 
Gladys Lechini • América Latina y África. Entre la solidaridad Sur-Sur y los propios...

la ruptura de relaciones diplomáticas con Sudáfrica.

La relación con Sudáfrica merece aquí una mención especial, pues a partir de 1994, luego de su acceso a la democracia multirracial, este país se convirtió en un socio importante para Brasil. Aunque el canciller Luiz Felipe Lampreia realizó tres viajes a Sudáfrica, la visita del presidente Cardoso en 1996, junto a varios ministros y una comitiva de setenta empresarios, marcó una diferencia importante al mostrar las intencionalidades de Brasil: aunar esfuerzos en las negociaciones multilaterales y desarrollar las potencialidades existentes, en los aspectos económicocomerciales. Del lado africano, las visitas a Brasilia confirman las preferencias brasileñas. Nelson Mandela visitó Brasil en 1991, en 1995 el canciller de la nueva Sudáfrica Alfred Nzo viajó a Brasilia, en 1997 fue el vicepresidente Tabo Mbeki y en 1998 volvió Nelson Mandela a Brasilia y a Buenos Aires para asistir a una cúpula presidencial del MERCOSUR, en Ushuaia. Finalmente, Tabo Mbeki estuvo en Brasilia, en diciembre de 2000, cuando volvió a cruzar el Atlántico para firmar en Florianópolis el Proyecto de Acuerdo Marco para la creación de un Área de Libre Comercio entre el MERCOSUR y Sudáfrica.

Con el ascenso de Luiz Inácio Lula da Silva a la presidencia en 2003, la dimensión africana volvió a tomar fuerza de la mano de Itamaraty y de otras agencias gubernamentales, en el contexto de una política pragmática y proactiva hacia los países del Sur, direccionada a la construcción de un liderazgo regional con inserción global. Varias acciones reflejan la decisión del ejecutivo y su coherencia entre política externa e interna. Ejemplos en el primer ámbito son el lanzamiento del Foro Brasil-África, en la ciudad de Fortaleza en junio de 2003 y la primera gira presidencial al África en noviembre de 2003. En el ámbito doméstico la política africana respondió a las crecientes demandas de los afrodescendientes, con la aprobación el 10 de enero de 2003 de la Ley Federal 10639 -que tornó obligatorio en todos los niveles de la enseñanza el estudio de la historia y la cultura africana y afrobrasileña- y con la creación el 21 de marzo de ese mismo año de la Secretaría Especial de Políticas de Promoción de la Igualdad Racial.

En paralelo, la actividad diplomática se intensificó a nivel bilateral y multilateral, a la vez que la relación comercial se incrementaba. Durante sus dos mandatos, el presidente realizó 11 giras por el continente, visitando 32 países y cerrando su gestión con un viaje a Mozambique junto a la presidenta electa, Dilma Rousseff. Esta fue una clara señal de continuidad de la política brasileña, confirmada con la gira africana de Rousseff, en 2011 y los 3 viajes realizados a lo largo de 2013, participando en las celebraciones de la Unión Africana.

Cabe remarcar que con la creación del Instituto Lula, el ex presidente continuó apoyando y profundizando 
el acercamiento africano a través de la África Initiative. En ese contexto se debe señalar el Seminario "Nuevos abordajes para erradicar el hambre en África hasta 2025", realizado entre el 30 de junio y el 1 de julio de 2013, en Addis Abeba, sede de la Unión Africana, junto a representantes de la Empresa Brasileña de Investigación Agropecuaria (Embrapa) y de la FAO.

Durante sus dos mandatos, la intensa actuación de Lula tuvo su correlato en el dinamismo diplomático desplegado a través de la apertura de 19 nuevas embajadas en África, de un total de 34. Del mismo modo, aumentaron la cantidad de convenios internacionales firmados con los países africanos, los cuales ascendieron a 346, un $67 \%$ de los 519 firmados en el período 19602010.

En materia de acción multilateral, es importante rescatar la promoción de nuevas iniciativas, como el foro IBSA y las ya mencionadas cumbres ASA y ASPA, así como la intensificación de las actividades con la Comunidad de Países de Lengua Portuguesa, entre las cuales figura la creación en 2010 de la UNILAB (Universidad de Integración Internacional de Lusofonía Afrobrasileña) en la ciudad de Redenção, estado de Ceará.

En cuanto a la cooperación técnica, que es gestionada por la Agencia Brasileña de Cooperación (ABC) desde 1987 , son datos destacables que el $50 \%$ de los gastos de ejecución se destinan a proyectos desarrollados en África. Las principales áreas temáticas son agricultura, lucha contra el hambre, preservación del medio ambiente y salud pública. Las actividades se desarrollan a través de empresas públicas como Embrapa o la Escuela Nacional de Salud Pública "Fundação Oswaldo Cruz" (Fiocruz).

Los datos del intercambio comercial también reflejan la creciente vinculación. Baste señalar que en diez años las exportaciones brasileñas aumentaron de 1.347.098.183 dólares en 2000, a 9.261.599.799 dólares en 2010 y que las importaciones desde África registraron valores de 2.907.082.676 dólares en el año 2000 a 11.302.224.181 en el $2010^{14}$. Estos números tienen lugar en una balanza comercial con una marcada presencia de commodities que han visto incrementados sus precios en la última década.

Para 2010, el 10\% de las transnacionales brasileñas se asentaba en África. Estas empresas se dedican principalmente a los sectores minero y de la construcción civil y están instaladas en 22 países africanos. Junto a la empresa petrolera estatal Petrobras, merecen mencionarse, entre otras, las constructoras Odebrecht, Mendes Júnior y el Grupo Camargo Correia; en tanto Vale do Rio Doce, multinacional líder en la producción de mineral de hierro y de níquel, está presente en siete países africanos, siendo Sudáfrica el socio más relevante.

Cabe mencionar que el sector privado brasileño ha recibido un gran

14 Fuente: Ministério do Desenvolvimento, Indústria e Comércio Exterior, 2011. 
Gladys Lechini • América Latina y África. Entre la solidaridad Sur-Sur y los propios...

apoyo de instituciones nacionales, principalmente del Banco Nacional de Desarrollo Económico y Social (BNDES), que ha estimulado las inversiones y exportaciones a través de préstamos. El ambiente de negocios también fue promovido por la Agencia Brasileña de Exportación (APEX), que fomenta la expansión de pequeñas y medianas empresas, a través de eventos como ferias comerciales y misiones de negocios, y la Caja Económica Federal (CEF).

Es también importante notar que a lo largo de la última década las inversiones extranjeras directas (IED) brasileñas en África crecieron de $69 \mathrm{mil}$ millones de dólares en 2001 a 214 mil millones en 2009. No obstante, de este total, la región subsahariana recibe una pequeña parte (124 millones de dólares en 2009), siendo Angola y Sudáfrica los principales receptores (IPEA, 2011: 86).

\section{Argentina Y LOS ImPUlsos AFRICANOS}

La política exterior argentina hacia los Estados del continente africano muestra un patrón de relaciones marcado por la dinámica de los impulsos, generando una relación espasmódica (Lechini, 2006:43-48), con altos (los impulsos) y bajos (la inercia y la no política).

Durante los impulsos se abrieron embajadas, se enviaron y recibieron misiones diplomáticas y comerciales y se incrementó el comercio. Sin embargo este conjunto de acciones no generó una masa crítica que promoviera el diseño de estrategias para los Estados de África, porque los impulsos respondieron a iniciativas puntuales, que luego se desvanecieron.

Factores propios de la inestabilidad política argentina, de la consiguiente orientación de su política exterior, las mudanzas en el sistema internacional y la particular situación de los países africanos actuaron como elementos condicionantes de la baja y errática vinculación externa de Argentina con estos países. Los cambios entre gobiernos civiles y militares contribuyeron a las fluctuaciones de la política exterior argentina y promovieron la política por impulsos. Pero la orientación del régimen no incidió fuertemente en su contenido, predominantemente comercial, salvo durante el gobierno de Alfonsín y el primer impulso con la presidencia de Arturo Frondizi, en que se combinaron con objetivos políticos. Los modos en que los diferentes y sucesivos gobiernos diseñaron la política exterior, y la poca y variable relevancia otorgada a las relaciones Sur-Sur, moldearon el bajo perfil de las relaciones argentino-africanas, tanto a nivel del discurso como de las acciones.

La baja prioridad de los Etados africanos en las políticas implementadas por los sucesivos gobiernos llevó a que las decisiones fueran tomadas a nivel del funcionamiento de "rutina" del Palacio San Martín. Generalmente las iniciativas de aproximación bilateral o multilateral (en el marco de los No Alineados y de las Naciones Unidas) 
se debieron a la buena voluntad e imaginación de los funcionarios a cargo del área, quienes lograban un espacio de maniobra para promocionar una acción u organizar una misión. Sin embargo, en el marco de una estructura desagregada, no necesariamente actuaron en forma coordinada y menos aún como parte de un diseño organizado sobre la base de elementos racionales y oportunidades a largo plazo. Solo la ruptura (1986) y el restablecimiento (1991) de relaciones diplomáticas con Sudáfrica fueron tomados en la cúspide del proceso decisorio.

El primer antecedente que mostró el interés por una aproximación al África fue el envío en 1960 del entonces embajador argentino ante las Naciones Unidas, Mario Amadeo, al festejo de las independencias de Zaire y de Somalia. De este modo se comenzó a gestar el primer impulso africano, el cual conllevó la elaboración del "Plan de presencia argentina en África”, que aconsejaba ya en 1961 (cuando solo 27 Estados africanos eran independientes) orientar la mirada argentina hacia ese continente. Este plan es fundacional, pues sus objetivos, presentados tan temprano, pueden considerarse subyacentes en casi todos los impulsos de esta relación errática. Estos mismos objetivos inspiraron en 1962 la misión de Juan Llamazares, que por entonces recorrió ocho países africanos. El segundo impulso se produjo en 1965, durante el gobierno de Arturo Illia, cuando el embajador Carlos Alberto Leguizamón presidió otra misión es- pecial al continente africano. En esta década se crearon varias embajadas: Sudáfrica, Marruecos, Nigeria, Ghana, Argelia, Senegal, Liberia y Etiopía. El tercer impulso fue la misión comercial a cargo de los consejeros Ramiro Arias y Rodolfo Potente, en 1974, durante la presidencia de Juan Domingo Perón.

Durante el gobierno militar, autodenominado Proceso de Reorganización Nacional (1976-1983), se produjo el cuarto impulso, con varias misiones comerciales que firmaron los primeros acuerdos de cooperación con países del África Negra, en el marco de una preocupación dominante en la política exterior argentina de la época: el cierre de los mercados tradicionales por las políticas proteccionistas de subsidios de la Comunidad Económica Europea. África fue así percibida como un mercado alternativo.

En 1982, con la crisis de las Malvinas, los Estados africanos que hasta entonces habían sido considerados marginales en la política exterior argentina, recibieron varias misiones políticas de buena voluntad e invitaciones a los jefes de Estado para visitar Buenos Aires, para conseguir su apoyo en la Asamblea General de las Naciones Unidas cuando se tratara el asunto Malvinas. Este quinto impulso convirtió a los Estados africanos en objeto de "uso diplomático".

Así como la práctica diplomática regular muestra que votos africanos y mercados aparecen subyacentes a los impulsos, existe otro tema que podría ser considerado una constante hasta la 
Gladys Lechini • América Latina y África. Entre la solidaridad Sur-Sur y los propios...

década de 1980 , referido a la república blanca y racista de Sudáfrica. Buenos Aires mantuvo una política dual para Sudáfrica y una política ambigua para con los países africanos en relación con el régimen del apartheid. La política dual consistió en mantener un discurso y una acción multilateral condenatorios del apartheid y buenas relaciones bilaterales con el gobierno blanco sudafricano. La política ambigua procuraba evitar tomar actitudes radicales con Pretoria por su política racista.

Pero durante el gobierno de Raúl Alfonsín, la ruptura de relaciones diplomáticas con Sudáfrica el 22 de mayo de 1986 marcó el fin de las ambigüedades entre los países de la llamada África Negra y Sudáfrica. Constituyó el sexto impulso, que apuntaba a consolidarse como política, al desarrollar una serie de acciones que se enmarcaban en un diseño de política exterior donde los No Alineados y el tercer mundo tenían un lugar. Entre los gestos se destacan los viajes argentinos a nivel presidencial y ministerial, tal el de Alfonsín a Argelia en octubre de 1984 -que se constituyó en la primera visita oficial de un jefe de Estado argentino al África- y su asistencia a la cumbre de los No Alineados, en Zimbabwe (Harare, septiembre de $1986)^{15}$. También se enviaron y reci-

15 Dante Caputo fue el primer canciller que realizó un viaje por África Subsahariana en 1988, visitando Angola, Costa de Marfil, Ghana, Gabón y Cabo Verde. El canciller otorgó relevancia política a los países africanos tanto en las instancias bilaterales como en las multilaterales. Visitó Argelia (diciembre de 1984) y Ma- bieron misiones de cooperación científica y tecnológica ${ }^{16}$ y se incrementó la relación comercial. Este fue el impulso más intenso con objetivos políticodiplomáticos y comerciales, elaborándose esta vez un diseño que comenzó a implementarse, pero que se desvaneció por la falta de continuidad durante la administración que lo sucedió.

Durante la gestión de Carlos Menem, a lo largo de los años noventa, no hubo estrategias para África: fue el momento más alto de la "política de la no política”. La relación diplomática se restringió a aquellos países con los cuales Argentina mantenía una vinculación comercial constante y montos importantes, o a Estados con potencialidades, como Nigeria o Sudáfrica. Así, se mantuvo una vinculación directa entre el perfil político-diplomático y el enfoque pragmático de las relaciones

rruecos (1985), y estuvo en Angola en 1985, en la reunión preparatoria de la cumbre de los No Alineados, y en Harare (1986). Asimismo habló en nombre del Grupo de los Ocho en Addis Abeba, en el aniversario de la creación de la Organización para la Unidad Africana (OUA), el 25 de mayo de 1988, frente a todos los representantes del continente africano.

16 El gobierno consideraba a la CTPD una parte integral de la política exterior del Estado y por ello en 1986 se dirigieron a los Estados africanos dos misiones de cooperación, cinco en 1987 y dos en 1988. Asimismo en 1987 y 1988 se realizaron en Argentina cuatro seminarios argentinoafricanos contando con el apoyo de organismos nacionales altamente capacitados como el Instituto Nacional de Tecnología Agropecuaria (INTA) y el Instituto Nacional de Tecnología Industrial (INTI) 
externas, con una balanza comercial favorable a la Argentina. Se cerraron las embajadas en Etiopía, Costa de Marfil, Gabón, Senegal y Zaire.

Pero a este declive del perfil político en los países del África Negra debe contraponerse un dato no menor: el restablecimiento de relaciones diplomáticas con Sudáfrica el 8 de agosto de 1991, y la consiguiente reapertura de la embajada argentina. Este séptimo impulso se reafirmó con el viaje de Menem a Sudáfrica el 24 de febrero de 1995, que lo constituyó en el primer mandatario americano en visitar oficialmente la flamante democracia. Una misión comercial multisectorial presidida por el embajador Eduardo Sadous en mayo de 1999 a Angola, Mozambique y Costa de Marfil, cerró la gestión.

La profunda crisis política y económica que vivió la Argentina a principios del nuevo siglo llevó a descuidar tanto el diseño como la implementación de la política exterior, que en el mejor de los casos fue reactiva. En ese contexto, se continuó con el enfoque pragmáticocomercialista, manteniendo las relaciones con los Estados del norte de África y Sudáfrica, con una balanza comercial altamente favorable a la Argentina.

El dato nuevo lo constituye el avance conjunto en las relaciones de Argentina y Brasil con los Estados del área, pudiendo hablarse ahora de un "impulso multilateral" en el sentido de las negociaciones entre el Mercosur y
$\mathrm{SACU}^{17}$, que tuvieron un hito en Florianópolis en 2000 y avanzaron con la firma, el 16 de diciembre de 2004 en Belo Horizonte, Brasil, de un acuerdo preferencial de comercio, un protocolo para la solución de controversias y un memorándo de entendimiento.

Durante la primera década del siglo XXI parece comenzar a gestarse un nuevo impulso con iniciativas tendientes a facilitar la relación bilateral, tales como la apertura o reapertura de embajadas (en Angola, Mozambique y Etiopía) y la firma de acuerdos. Aquí es importante subrayar que mientras que en el período 1960-2003 se firmaron 88 actos internacionales, entre 2003 y 2011 se suscribieron 70 acuerdos $^{18}$, lo cual es un indicador de la mayor institucionalización de los vínculos y de los intereses (Morasso,2012).

Sin embargo y a pesar de los acuerdos y de la visita de los ministros de relaciones exteriores y funcionarios de nivel en el continente, entre 2003 y 2012 solamente hubo 2 viajes presidenciales por África. En 2008 la presidenta Cristina Fernández visitó

17 La Unión Aduanera del África Austral está integrada por Sudáfrica, Namibia, Lesotho, Swazilandia y Botswana

18 Los Estados con los cuales se firmaron acuerdos fueron Argelia, Angola, Egipto, Guinea Ecuatorial, Libia, Marruecos, Mozambique, Namibia, Nigeria, Sudáfrica, Túnez y Zambia. Es de destacar que esta lista incluye también los MOU (Memorandum of Understanding) o cartas de intención, que expresan una convergencia de deseo entre las partes y son más informales que un acuerdo 
Gladys Lechini • América Latina y África. Entre la solidaridad Sur-Sur y los propios...

África Nordsahariana ${ }^{19}$ en una misión comercial multisectorial en el marco de la cual se organizaron reuniones políticas bilaterales y se firmaron acuerdos de cooperación en agricultura, tecnología, comercio e inversiones, desarrollo social, ganadería y pesca. En 2012, la primera mandataria llegó a Luanda, Angola, acompañada de una nutrida comitiva de empresarios, para participar de la Feria Empresaria Argentina. Por otra parte, se recibieron en Buenos Aires los presidentes de Angola y Guinea Ecuatorial y autoridades de alto rango como ministros y cancilleres.

En cada uno de estos encuentros bilaterales al igual que en los ámbitos multilaterales se destacó en el discurso la adscripción a la CSS. En las cumbres ASA y ASPA Argentina enfatizó que la coyuntura sistémica requiere estrechar el diálogo Sur-Sur, recuperando las coincidencias en las agendas regionales. En línea, se encuentran el ingreso de Argentina como observador en la Unión Africana (UA) en 2009 y en la Comunidad Económica de Estados de África Occidental (ECOWAS) en 2010.

Pero más allá de la retórica se ha avanzado en la cooperación técnica horizontal a través del Fondo Argentino de Cooperación Horizontal (FO-AR), creado en 1992, que desarrolla sus acciones principalmente en tres temas: administración y gobernabilidad; derechos humanos; y desarrollo sustentable.

Las acciones con los países africanos giraron en torno al apoyo al desarrollo

19 Estuvo en Argelia, Túnez, Libia y Egipto. productivo de los sectores agrícolas y de servicios que promuevan la preservación de los recursos naturales ${ }^{20}$. Esta actividad pudo desenvolverse gracias a las fortalezas argentinas, a la expansión de la frontera agrícola africana y a la revaluación de los precios de los alimentos.

La organización por parte del Ministerio de Agricultura de la visita de ministros de agricultura africanos y funcionarios de organizaciones internacionales africanas en 2011 puso de manifiesto el interés argentino en estrechar vínculos con África en materia de agricultura y agroindustria que impliquen el traspaso de tecnología para incrementar la producción y la apertura de mercados para las empresas de maquinaria argentinas. El encuentro se enmarcó en la necesidad de establecer las líneas de cooperación estratégicas para el sector agroalimentario con vistas al futuro rol de África como proveedor de alimentos.

No es un dato menor la satisfactoria balanza comercial para Argentina que se mantiene a lo largo de los años. Si bien los flujos de intercambio representan una baja porción del comercio de Argentina con el mundo, en promedio un $6 \%$ en la última década, lo importante es el salto que el mismo tuvo: de 1.275.566 dólares en 2001

20 Cabe señalar que en muchas de las iniciativas de CSS intervienen también otros organismos nacionales especializados, tales como el INTI, el INTA y el Ministerio de Ciencia, Tecnología e Innovación Productiva. 
a 4.022.152 dólares en 2010. En el período 2005-2010 el intercambio comercial se duplicó, reportándose en 2010 un fuerte superávit: se exportaron al continente 4.022.152 millones de dólares y se importaron 336.99421.

Los principales socios son Angola, Egipto, Marruecos, Libia, Túnez, Sudáfrica, Argelia, Nigeria, Kenya y Mozambique, a los que se exportan principalmente materias primas, pero cada vez con un mayor componente de productos con valor agregado, tanto en productos de consumo terminados como maquinaria y equipo de transporte. En este sentido, los analistas destacan que es interesante el caso argentino porque a pesar de la supremacía de los productos oleaginosos, agropecuarios, cereales y lácteos, los productos industriales y los combustibles también están presentes, lo cual implica un cierto potencial para insertarse en el mercado africano. En cuanto a las importaciones desde África, se centran en combustibles minerales y productos químicos.

\section{VENEZUELA Y LA "PETRO- DIPLOMACIA” EN ÁFrICA}

La "revolución bolivariana" del presidente Hugo Chávez Frías marcó un quiebre con la tradicional política exterior venezolana que desde la década de 1960 tenía como eje rector las estrechas relaciones con Estados Unidos y los vínculos comerciales Norte-Sur.

21 Fuente: Instituto Nacional de Estadísticas y Censos de Argentina (INDEC).
Desde sus inicios en 1999, el gobierno chavista propuso una política exterior que fortaleciese la soberanía nacional, diversificando las relaciones externas y las redes de cooperación. En ese contexto promovió una solidaridad renovada con África haciendo uso, al igual que Brasil, del componente africano de su población. La estrategia africana comenzó a tomar forma en 2005, con un cambio institucional relevante, cual la creación del cargo de viceministro para África, nombrando a Reinaldo Bolívar, con la elaboración anual de la "Agenda África" y con el desarrollo de un activismo diplomático y presidencial significativo.

Uno de los datos más destacables es el establecimiento de relaciones diplomáticas con la totalidad de los 54 países del continente y la apertura de 10 nuevas embajadas ${ }^{22}$, que suman 18 en total. En la misma sintonía se suscribieron acuerdos para darle sustento jurídico a los vínculos incipientes. Según Reinaldo Bolívar, viceministro para África, entre 1957 y 2004 el país había firmado escasamente treinta acuerdos de cooperación con África, en tanto que para el 2010 se habían superado los 200 convenios firmados ${ }^{23}$. Por otra parte, Venezuela ingresó como observador a la UA, a la ECOWAS y a la SADC.

22 Se encuentran en Etiopia, Senegal, Mali, Gambia, Benín, Guinea Ecuatorial, Angola, Mozambique, Congo y Sudan.

23 En "Viceministro Reinaldo Bolívar presentó agenda África 2009", Aporrea, 8 de abril de 2009. Disponible en: http://www. aporrea.org/venezuelaexterior/n132491. html 
Gladys Lechini • América Latina y África. Entre la solidaridad Sur-Sur y los propios...

En cuanto a las giras presidenciales, la primera tuvo lugar en 2006, año en el cual Chávez viajó tres veces a África ${ }^{24}$. En 2008 visitó Sudáfrica; en 2009, asistió a la Cumbre de la UA en Libia y volvió a Argelia y en 2010 regresó a Libia. En cada viaje se suscribieron acuerdos de cooperación y comercio y se realizaron declaraciones políticas a favor del estrechamiento de los lazos Sur-Sur. Como correlato de las visitas del presidente venezolano arribaron a Caracas los mandatarios de Nigeria (2005); de Gambia, (2007) y el presidente de la UA. Pero fue la llegada de 25 jefes de Estado a la Isla Margarita para la II Cumbre ASA el mayor logro diplomático de Chávez.

En el marco de las acciones de CSS, se destacan las contribuciones de Venezuela a los organismos internacionales para el desarrollo africano, así como el aporte financiero al Programa Mundial de Alimentos (PMA) para paliar el hambre en Burkina Faso, Kenya, Mali, Mauritania, Níger, Somalia y Zimbabwe y a la Organización para la Alimentación y la Agricultura (FAO), destinados a apoyar proyectos de intensificación agrícola para el control del agua en Burkina Faso y Mali (Giacchi, 2011)

En materia cultural y educativa, el gobierno de Chávez ha realizado esfuerzos para revalorizar la herencia africana en la población venezolana y desplegar una "diplomacia de los pueblos". En el año 2005 se incorporó al calendario el día de la afrovenezolanidad y en 2011

24 Visitó Benin, Malí, Angola, Guinea Bissau, Cabe Verde, Senegal, Argelia y Libia. se incluyó en el censo de ese año la variable étnica-racial para afrodescendientes. Asimismo, se han organizado festivales culturales con los pueblos africanos, la Semana Mundial de África y las cátedras libres sobre África en el ámbito de las universidades.

La revalorización de la herencia africana es parte del proceso que impulsa al gobierno para aumentar los lazos de cooperación con el continente. En ese marco se lleva adelante desde 2007 el programa "Apadrina una Escuela en África" a través del Banco de Desarrollo Económico y Social, para asistir a escuelas con carencias. De acuerdo con los datos del Despacho del Viceministro para África, el programa se ha desarrollado en 16 países africanos beneficiando a más de 70 mil niños (Giacchi, 2011).

En consonancia con la fuerte impronta petrolera de la política exterior, entre 2004 y 2010 Venezuela firmó convenios en materia energética con 16 países africanos, principalmente a través del Ministerio del Poder Popular para la Energía y Petróleo. En ellos se plasma la intención de intercambiar información y expertos, cooperar en la explotación, producción, almacenamiento, transporte, refinación y distribución de petróleo y gas y emprender inversiones conjuntas. Durante el encuentro presidencial en 2008, Chávez y Mbeki firmaron un acuerdo energético que propone a la empresa petrolera de Sudáfrica (PetroSA) trabajar conjuntamente con Petróleos de Venezuela S. A. 
(PDVSA) en la exploración de la Faja Petrolífera del Orinoco.

El petróleo marca la agenda comercial venezolana y determina su inserción. La duplicación entre 2004 y 2008 de las exportaciones venezolanas al continente ${ }^{25}$ se debió a las exportaciones de petróleo. Sin embargo, las ventas de petróleo a África representan el $0,79 \%$ del total mundial exportado, frente a un $52 \%$ que se destina a Estados Unidos, por lo cual aún se necesita una mayor diversificación si Venezuela pretende disminuir su dependencia y ampliar sus márgenes de acción.

\section{REFLEXIONES FINALES}

Desde la independencia de los países africanos, los Estados de América Latina han desarrollado con estos nuevos actores una cooperación incipiente, con altibajos, muy dependiente de los imperativos internos y aprovechando los márgenes de maniobra del sistema internacional. La africanidad en América Latina, la solidaridad Sur-Sur y la búsqueda de una mayor autonomía fueron elementos que impulsaron el acercamiento hacia las otras costas del Atlántico.

Brasil ha mantenido una política africana a lo largo de los años, profundizando el enfoque a partir de la gestión de Lula da Silva y utilizando la "diplomacia cultural" y la coopera-

25 Fuente: Banco de Comercio Exterior (BANCOEX) de la República Bolivariana de Venezuela. ción Sur-Sur como paraguas protector. Argentina ha desarrollado una política impulsiva, con un tinte comercialista, aunque nunca llegó a sostener una estrategia de acercamiento que perdurase a lo largo de los años. Venezuela, por su parte, se convirtió con Chávez en la presidencia en el nuevo jugador africano, desplegando una "petro-diplomacia" que tendrá límites muy precisos.

Entre el acercamiento bilateral y las estrategias multilaterales y regionales, los Estados de América Latina y de África se están conectando en un mundo que está lentamente cambiando, a partir de una nueva reconfiguración de fuerzas. Para optimizar su inserción internacional necesitan desarrollar nuevas estrategias en un contexto de nuevas realidades y buscando marcos conceptuales y analíticos propios, sobre la base del conocimiento construido, adquirido y compartido desde diferentes latitudes.

Pero en este acercamiento, el discurso solidario no siempre tuvo ni tiene su correlato en acciones cooperativas debido a intereses divergentes, como también a imperativos internos y sistémicos. El caso de Brasil es el ejemplo más claro, aunque puede hacerse extensivo a los demás. Si bien muestra a nivel discursivo un compromiso Sur-Sur y un despliegue de acciones que algunos califican de "fuegos de artificio", es aún un país con una economía emergente que debe saldar deudas sociales y compatibilizarlas con los compromisos externos, así como evitar contradicciones entre discurso y práctica. 
Gladys Lechini • América Latina y África. Entre la solidaridad Sur-Sur y los propios...

Una mejora en la inserción internacional de los países de América Latina y de África redundará en beneficio de sus pueblos. La cooperación Sur-Sur puede ser una alternativa complementaria a la diversificación de las relaciones externas y la búsqueda de nuevos socios, en múltiples juegos y alianzas. Sin embargo, los gobiernos de nuestros países deben estar a la altura de las circunstancias para poder dar las respuestas apropiadas a los desafíos que presenta un sistema internacional con fuertes turbulencias y apuntar a construir un mundo incluyente y mejor distribuido.

\section{BibliogRAFIA}

Barber, James (2004), Mandela's World. The International Dimension of South Africa's Political Revolution 1990-99, Cambridge University Press.

Bolívar, Reinaldo (2007), "Venezuela-África, las vueltas de la historia", en Política Exterior y Soberanía, año $2, n^{\circ} 4$, Instituto de Altos Estudios Diplomáticos "Pedro Gual", Venezuela.

Bolívar, Reinaldo (2011), Entrevista, en ENcontrARTE, $n^{\circ} 142$, Aporrea, Venezuela.

Brun, Elodie (2008), Las relaciones entre América Latina y África ¿Potenciales o ilusorias?, Colección Documentos de Proyecto, CEPAL, Santiago de Chile.

Brasil. Ministério das Relaçoes Exteriores do Brasil. (s.f.). Exposição do Embaixador Luiz F Lampreia, Ministro de Estado das Relações Exteriores, no X Fórum Nacional, As perspectivas da Situação mundial e a Política Externa do Brasil. Recuperado el 12 de mayo de 1998 de http:///w.w.w.mre. gov.br/sei/disforum/htm.

Costa Leite, Iara (2012), Cooperação Sul-Sul: conceito, história e marcos interpretativos, en Observador On-Line, Observatorio
Político Sul-Americano, IESP/UERJ, v.7, n.3, marzo, Río de Janeiro.

Delgado Caicedo, Jerónimo y A. Barrera (2010) Subiendo al Sur: África y América Latina en las nuevas dinámicas de la cooperación sur-sur, en Humanía del Sur, 5, enero-junio, Universidad de Los Andes, Mérida.

Fonseca, Olga (2007), Evolución de las relaciones diplomáticas Venezuela-África 1999-2007, en Política Exterior y Soberanía, año 2, n ${ }^{\circ}$, Instituto de Altos Estudios Diplomáticos "Pedro Gual", Venezuela.

Giacchi Maria Florencia (2011), La política exterior del gobierno de Hugo Chávez hacia África en el período 2004-2010. (Tesis de doctorado en relaciones internacionales) Universidad Nacional de Rosario.

Instituto de Pesquisa Econômica Aplicada y Banco Mundial (2011) Ponte sobre o Atlantico. Brasil e Africa Subsaariana. Parceria Sul-Sul para o Crescimento, Brasilia www.ipea.org.br

Keet, Dot (2006), South-South Strategic Alternatives to the Global Economic System and Power Regime, Amsterdam: Transnational Institute.

Kern, Alejandra y Lara Weisstaub (2011), El debate sobre la cooperación sur-sur y su lugar en la política exterior argentina, en Revista Española de Desarrollo y Cooperación, Instituto Universitario de Desarrollo y Cooperación, $\mathrm{N}^{\circ} 27$, invierno, Madrid.

Lampreia, Luiz Felipe (1995, Marzo 11). Calendário da Diplomacia Presidencial em 1995, en O Globo.

Lander, Edgardo (comp.) (2000), La colonialidad del saber: eurocentrismo y ciencias sociales. Perspectivas latinoamericanas, Buenos Aires: CLACSO.

Lechini, Gladys (1986), Así es África. Su inserción en el mundo. Sus relaciones con Argentina, Buenos Aires: Editorial Fraterna.

Lechini Gladys (1995), Las relaciones Argentina-Sudáfrica desde el Proceso hasta Menem, Rosario: Ediciones CERIR.

Lechini, Gladys (2006), Argentina y África en el espejo de Brasil. ¿Política por impulsos 
o construcción de una política exterior?, Buenos Aires: CLACSO.

Lechini, Gladys (2007), "IBSA: Una opción de cooperación Sur-Sur”, en Girón, Alicia, Correa, Eugenia (ed.), Del Sur hacia el Norte: Economía política del orden económico internacional emergente, Buenos Aires: CLACSO, pp. 271-285.

Lechini, Gladys (2009) La cooperación SurSur y la búsqueda de autonomía en América Latina ¿Mito o realidad?, en Revista Relaciones Internacionales, Universidad Autónoma de Madrid, Madrid, número 12, Págs. 55 a 81.

Malacalza, Bernabé (2013) Estrategia y experimentalismo. Los procesos domésticos de configuración de la Cooperación Sur-Sur desde una perspectiva macro-micro. Un estudio comparado sobre los casos de la Argentina y Brasil (2003-2011), tesis doctoral, Doctorado en Ciencias Sociales, Facultad Latinoamericana de Ciencias Sociales (FLACSO), Sede Académica Argentina, Buenos Aires.

Morasso, Carla, 2012, Las negociaciones MERCOSUR-África Austral. Avanzando en los vínculos Sur-Sur, Editorial Académica Española, Berlín.

Mourão, Fernando A. Albuquerque (1994), "O Brasil e a Africa”, en Fonseca Júnior, Gelson, Nabuco de Castro, Sergio Henrique (org.), Temas de Política Externa Brasileira II, São Paulo: Paz e Terra,

Nunes Pereira, Luena (2008) “O ensino e a pesquisa sobre Africa no Brasil e a Lei 10639" en Lechini, Gladys (comp), Los estudios afroamericanos y africanos en América Latina. Herencia, presencia y visiones del otro, Córdoba: CLACSO/ Programa de Estudios Africanos (CEA),

Penna, Pio (2001, octubre), A evolução das relações entre o Brasil e a África do Sul - de 1918 a 2000. Ponencia presentada en el $\mathrm{X}$ Congresso Internacional da ALADAA, Rio de Janeiro, Brasil.

Pereira, José Maria Nunes y Edson Borges (1992, agosto), O imperativo da política africana do Brasil. Ponencia presentada en el II Congresso Luso-Brasileiro de Ciências Sociais, São Paulo, Brasil.

Rizzo Romano, Alfredo (1991), “Misión argentina en África” Buenos Aires, Revista Argentina Ante el Mundo.

Said, E. (1996), Culture and Imperialism, Nueva York, Vintage.

Saraiva, José Flavio Sombra (1996), O lugar da África. A dimensão Atlântica da Politica Externa Brasileira (de 1946 a nossos días), Brasilia: Editora UNB.

Saraiva, José Flavio Sombra (2002), "Política exterior do Governo Lula; o desafío africano”, RBPI, Ano 45, No 2.

Souza A. A agenda Internacional do Brasil: um estudo sobre a Comunidade Brasilerira de política Externa (2002) (mimeo) Rio de Janeiro, CEBRI, en Soares de Lima, Maria Regina, A política externa brasileira e os desafios da cooperaçao Sul- Sul, RBPI, Brasilia, Ano 48, No1, 2005

Vilalva, Mário e Irene Vida Gala (2001), "Relações Brasil-África do Sul: quatro décadas rumo à afirmação de uma parceria democrática (1948-1998)", Cena Internacional, Año 3, $\mathrm{N}^{\circ} 2$.

Worsley, Peter (1972), El Tercer Mundo una nueva fuerza vital en los asuntos internacionales, Buenos Aires: Siglo Veintiuno Editores.

Zuluaga Nieto, Jaime (2006), A tricontinental of Knowledge: a Space for South-South Co-operation, en Boron Atilio y Gladys Lechini (eds.) (2005), Politics and social movements in an hegemonic world. Lessons from África, Asia and Latin America, Buenos Aires, Ediciones CLACSO, p. 402.

\section{Pags web}

"Chávez ofreció discurso en cumbre de la Unión Africana en Trípoli”, Noticias 24 (31/8/2009). Disponible en: http://www. noticias24.com/actualidad/noticia/80172/ chavez-interviene-en-la-cumbre-de-launion-africana-en-tripoli/ [Consultado el $1 / 9 / 2011]$ 
Gladys LeChini • América Latina y África. Entre la solidaridad Sur-Sur y los propios...

"En 6 años se han duplicado las relaciones diplomáticas con África”, Prensa YVKE, Ministerio del Poder Popular para la Comunicación y la Información de Venezuela (14/1/2011). Disponible en: http:// www.radiomundial.com.ve/yvke/noticia. php?p=405483 [Consultado el 2/9/2011]

Entrevista a Reinaldo Bolívar, Ministerio del Poder Popular para la Comunicación y la Información de Venezuela, (22/9/2009). Disponible en: http://www.alopresidente. gob.ve/informacion/3/1380/reinaldo_ bolunvar $\%$ E2 $\% 80 \% 9 \mathrm{Cu} \% 20$ frica_bienvenida.html [Consultado el 31/8/2011]

"Presidente Chávez: 'África tiene todo para ser un polo de poder'”, Prensa Presidencial, Ministerio del Poder Popular para la Comunicación y la Información (1/7/2006). Disponible en: http://www.minci.gob. ve/noticias-prensa-presidencial/28/9778/ presidente_chez $\%$ E2\% 80\%9Crica_tiene. html [Consultado el 1/9/2011] 